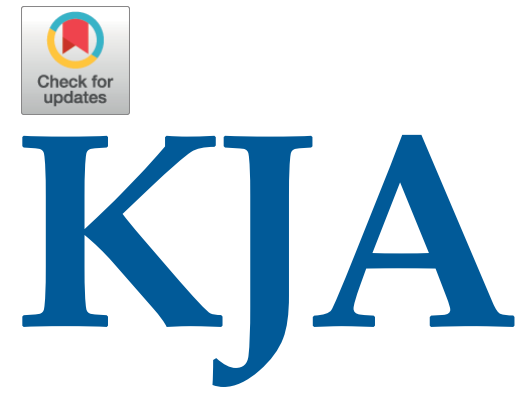

Korean Journal of Anesthesiology

\section{Letter to the Editor}

Korean J Anesthesiol 2020;73(2):173-174

https://doi.org/10.4097/kja.20013

pISSN 2005-6419 • eISSN 2005-7563

Received: January 11, 2020

Accepted: January 28, 2020

Corresponding author:

Anju Romina Bhalotra, M.D.

Department of Anesthesiology, Maulana Azad

Medical College and Associated Lok Nayak

Hospital, A-1/59 Safdarjang Enclave, New

Delhi 110029, India

Tel: +91-01126183684

Email:drakgk@yahoo.co.in

ORCID: https://orcid.org/0000-0003-0700-2941

(C) The Korean Society of Anesthesiologists, 2020

(c) This is an open-access article distributed under the terms of the Creative Commons Attribution Non-Commercial License (http://creativecommons.org/licenses/ by-nc/4.0/), which permits unrestricted non-commercial use, distribution, and reproduction in any medium, provided the original work is properly cited.

\title{
Awake supraglottic airway guided flexible bronchoscopic intubation
}

\section{Anju Romina Bhalotra}

Department of Anesthesiology, Maulana Azad Medical College and Associated Lok Nayak Hospital, New Delhi, India

I read with great interest the narrative review on awake supraglottic airway guided flexible bronchoscopic intubation in patients with anticipated difficult airways by Lim and Wong [1]. Undoubtedly, awake intubation is indicated in a patient when difficulty in maintaining or securing the airway after induction of general anesthesia is expected. The natural airway is better maintained in the awake patient. The normal muscle tone helps to maintain the anatomy and easier identification of upper airway structures relative to each other. There is less likelihood of aspiration with preservation of the lower esophageal sphincter tone and maintenance of spontaneous breathing; besides, the patient can follow instructions while awake. However, awake intubation requires careful patient preparation, adequate topical anesthesia of the airway, and judicious use of sedative agents; besides, an appropriate level of operator expertise. The insertion of a supraglottic airway device (SAD) seated in the pharynx above the laryngeal inlet attenuates the pressor and laryngeal responses [2]. It is reasonable to assume that in the awake patient, an SAD may be better tolerated than an endotracheal tube. The SAD serves as a conduit for fiberoptic bronchoscope-guided endotracheal intubation and, if positioned correctly, leads to easier and more rapid endotracheal intubation. The Difficult Airway Society guidelines for the management of the unanticipated difficult airway [3] and the American Society of Anesthesiologists Difficult Airway algorithm [4] suggest intubation through an SAD in case of failed tracheal intubation. Insertion of an SAD in the awake patient under topical anesthesia followed by tracheal intubation after induction of general anesthesia has been reported previously [3]. In their narrative review, Lim and Wong describe inserting an Ambu Auragain ${ }^{\mathrm{TM}}$ SAD after topical anesthesia of the airway and remifentanil infusion. Fiberoptic bronchoscope-guided tracheal intubation was achieved through the SAD. However, it is not clear why the authors did not inflate the SAD cuff. The position of an SAD is usually confirmed after cuff inflation. Cuff inflation permits the formation of a seal between the device and the pharyngeal mucosa; besides, the use of a second-generation SAD also allows isolation of the respiratory and alimentary tracts. Cuff inflation also prevents device displacement. There seems to be no recommendation in literature to leave the SAD cuff uninflated. Furthermore, insufflation of oxygen at $10-15 \mathrm{~L} / \mathrm{min}$ through an oxygen tubing attached to the proximal end of the ventilation port of the SAD may lead to gastric insufflation, regurgitation, and pulmonary aspiration. The authors report supraglottic airway guided flexible bronchoscopic intubation in several cases. However, when coughing or patient movement during intubation is undesirable, as in patients with intracranial lesions or an unstable cervical spine, it may be prudent to administer general anesthesia with or without the use of muscle relaxants after confirmation of SAD position by fiberoptic bronchoscopy. This may be followed by assisted ventilation before attempting tracheal intubation through the SAD. 


\section{Conflicts of Interest}

No potential conflict of interest relevant to this article was reported.

\section{References}

1. Lim WY, Wong P. Awake supraglottic airway guided flexible bronchoscopic intubation in patients with anticipated difficult airways: a case series and narrative review. Korean J Anesthesiol 2019; 72: 548-57.

2. Oczenski W, Krenn H, Dahaba AA, Binder M, El-Schahawi-Kienzl I, Jellinek H, et al. Hemodynamic and catecholamine stress responses to insertion of the Combitube, laryngeal mask airway or tracheal intubation. Anesth Analg 1999; 88: 1389-94.
3. Freck CM, Mitchell VS, McNarry AF, Mendonca C, Bhagrath R, Patel A, et al. Difficult Airway Society 2015 guidelines for the management of unanticipated difficult intubation in adults. Br J Anaesth 2015; 115: 827-48.

4. Apfelbaum JL, Hagberg CA, Caplan RA, Blitt CD, Connis RT, Nickinovich DG, et al. Practice Guidelines for Management of the Difficult Airway: An Updated Report by the American Society of Anesthesiologists Task Force on Management of the Difficult Airway. Anesthesiology 2013; 118: 251-70.

5. Ludeña JA, Bellas JJA, Rementeria RA, Muñoz Alameda LE. Assessment of awake i-gel ${ }^{\text {Tw }}$ insertion for fiberoptic-guided intubation in patients with predicted difficult airway: a prospective, observational study. J Anaesthesiol Clin Pharmacol 2018; 34: 490-5. 